\title{
Markers of liver function and inflammatory cytokines modulation by aerobic versus resisted exercise training for nonalcoholic steatohepatitis patients
}

\author{
Shehab M. Abd El-Kader'르, Osama H. Al-Jiffri², Fadwa M. Al-Shreef ${ }^{2}$
}

1. Department of Physical Therapy, Faculty of Applied Medical Sciences, King Abdulaziz University.

2. Department of Medical Laboratory Technology, Faculty of Applied Medical Sciences, King Abdulaziz University.

\begin{abstract}
Background: Non-alcoholic steatohepatitis is a growing public health problem with no approved therapy; as cytokines and other pro-inflammatory mediators may each play a role in transition of steatosis to NASH which is projected to be the leading cause of liver transplantation in the United States by 2020.

Objective: The aim of this study was to compare the impact of aerobic versus resisted exercise training on inflammatory cytokines and markers of liver function in patients with nonalcoholic steatohepatitis.

Material and Methods: Fifty patients with NASH were included in the study and divided into two subgroups. Participants were included into 2 equal groups; the first group (A) received aerobic exercise training. The second group (B) received resisted exercise training three times a week for 3 months.

Results: The mean values of TNF- $\alpha$, IL6, IL8, ALT and AST were significantly decreased in group (A) and group (B).Also; there was a significant difference between both groups after treatment.

Conclusion: Aerobic exercise training modulates inflammatory cytokine levels and markers of liver function in patients with nonalcoholic steatohepatitis.
\end{abstract}

Keywords: Aerobic Exercise; Resisted Exercises; Inflammatory Cytokine; markers of liver function; Nonalcoholicsteatohepatitis.

DOI: http://dx.doi.org/10.4314/ahs.v14i3.8

\section{Introduction}

Non-alcoholics steatohepatitis (NASH) is a chronic progressive liver disease characterized by accumulation of fat in the liver accompanied by necro-inflammation and hepatocellular injury $[1,2]$. NASH prevalence is estimated between $2-3 \%$ respectively of the general population in Western societies [3]. In all probability $\mathrm{NASH}$ prevalence figures will rise in the future as $\mathrm{NASH}$ is considered the hepatic manifestation of the metabolic syndrome and the number of overweighed individuals is growing. However, the exact mechanism of the progression from a benign steatotic liver to an inflamed organ remains a point of interest as NASH is projected to be the leading cause of liver transplantation in the United States by 2020 [4,5].

\section{Corresponding Author:}

Shehab Mahmoud Abd El-Kader

Department of Physical Therapy,

Faculty of Applied Medical Sciences,

King Abdulaziz University,

P.O. Box 80324, Jeddah, 21589, Saudi Arabia

Phone: +966-569849276

E.mail: salmuzain@kau.edu.sa
Nonalcoholic steatohepatitis (NASH) is a liver disease characterized by diffuse fatty acid infiltration and inflammation. The exact cause of NASH is unclear, but it is increasingly becoming more evident that the disease is much more common than was previously thought [6]. $\mathrm{NASH}$ is seen in patients of all ages, including children, and is associated with over nutrition and under activity, insulin resistance, and genetic factors. Lipotoxicity, oxidative stress, cytokines, and other pro-inflammatory mediators may each play a role in transition of steatosis to NASH $[7,8]$.

The pathogenesis of NASH includes insulin resistance, increased inflammation, tumor necrosis factor-alpha (TNF- $\alpha$ ), interleukin-6 (IL-6), interleukin-8 (IL-8) and increased oxidative damage $[5,9,10]$. In many cases, NASH causes asymptomatic elevation of the level of liver transaminases. Alanine aminotransferase (ALT) is most closely related to liver fat accumulation, and it has been used as an indicator of NASH [11]. Since liver biopsy cannot be performed as a screening method to detect $\mathrm{NASH}$ in general population, abdominal ultrasonography as a noninvasive modality has been widely used [12].

Two adipocyte-derived hormones, leptin and adiponectin, have been implicated in the pathogenesis of both 
non-alcoholicsteatohepatitis and insulin resistance. In subjects with congenital lipodystrophy syndrome and low serum leptin concentrations, severe steatosis and insulin resistance have been reported [13]. Treatment of these subjects with leptin reduced liver fat and liver enzyme abnormalities and improved insulin resistance. Low concentrations of adiponectin have been associated with high visceral fat and have been implicated in the development of the insulin resistance syndrome. Furthermore, in a study of 90 morbidly obese Chinese subjects, plasma concentrations of adiponectin were inversely correlated with those of ALT [14].

Leptin induces the release of pro-inflammatory cytokines such as TNF- $\alpha$ which amplifies the process [15]. If the balance is not restored the protective mechanisms of the adipose tissue become harmful and the body is entering a chronic inflammatory state, which is particularly seen in central obesity [16].

Adiponectin protects from inflammation and fibrosis in metabolic liver disease. Adiponectin is an adipose tissue released protein which ameliorates insulin resistance, and thereby, may protect from NAFLD. However, systemic levels are reduced in these patients $[17,18]$. In animal models of non-alcoholicsteatohepatitis (NASH) adiponectin lowers inflammation, reactive oxygen species production and liver fibrosis and thereby, may protect from progressive liver disease $[14,19]$.

Few studies have prospectively examined the effect of exercise on elevated levels of inflammatory biomarkers in diabetic subjects and found contrasting results in terms of efficacy and dependence on weight loss [20,21]. The potential mechanisms for the anti-inflammatory effect of exercise, including reduced percentage of body fat and macrophage accumulation in adipose tissue, muscle-released interleukin- 6 inhibition of tumor necrosis factor-a, and the cholinergic anti-inflammatory pathway [22].

The aim of this study was to compare the impact of aerobic versus resisted exercise training on inflammatory cytokines and markers of liver function in patients with nonalcoholic steatohepatitis.

\section{Material and methods \\ Subjects}

Fifty NASH patients (26 men, 24 women; mean age of $51 \pm 6$ years) with body mass index (BMI) ranged from
31 to $36 \mathrm{Kg} / \mathrm{m}^{2}$, were selected from gastroenterology outpatient clinic, King Abdulaziz University Hospital, Jeddah, Saudi Arabia. Subjects were excluded if they did not agree to participate, had a history of diabetes mellitus, smoking, cardiovascular disease, had alcohol abuse problems, hepatic (i.e. infectious and viral disease) or renal disease, and if they were being treated with antihyperlipidemic, antihypertensive or any antioxidants like vitamin C or E medications. Participants were included into 2 equal groups; the first group (A) included 13 men and 12 women who received moderate aerobic exercise training. The second group (B) included 13 men and 12 women who received mild aerobic exercise training three times a week for 3 months. The study was approved by the Ethics Committee of King Abdulaziz University Hospital, Jeddah, Saudi Arabia and all patients gave informed consent for participation in this study. If any adverse effects had occurred, the experiment would have been stopped. However, no adverse effects occurred, and so the data of all the participants was available for analysis.

Each patient underwent abdominal US (Siemens Antares equipment with $\mathrm{CH} 4-1 \mathrm{MHz}$ transducer; Siemens Medical, Erlangen, Germany) in a fasting state. The presence of hepatic steatosis was assessed independently by radiologists according to findings such as hepatorenal contrast, blurring of the vascular wall, and profound attenuation of the diaphragm. According to the findings of ultrasound examination patient group with NASH was divided into three; as minimal, moderate and marked subgroup parallel to the increase in echogenicity [23].

\section{Laboratory analysis}

Venous blood samples were collected in polystyrene tubes after a 12 hours fasting by venipuncture of the antecubital vein while patients rested in a supine position. The blood samples were transported to a laboratory within 1 hour and centrifuged at $4^{\circ} \mathrm{C}$ to remove serum. Serum ALT, AST levels were assayed using a Cobas Integra 800 analyzer (Roche Diagnostics, Geneve, Switzerland). While IL-6 levels were measured using "Immulite 2000" immunassay analyzer (Siemens Healthcare Diagnostics, Deerfield, USA). However, interleukin-8 (IL-8) and TNF- $\alpha$ levels were analyzed with ELISA kits using ELISA microplate strip washer (ELX 50), and ELISA microplate reader (ELX 808; BioTek Instruments, USA).

\section{The aerobic exercise training program}

Patients in Group A were submitted to a 40 min aerobic 
session on a treadmill (the initial, 5-minute warm-up phase performed on the treadmill at a low load, each training session lasted 30 minutes and ended with 5-minute recovery and relaxation phase) either walking or running, based on heart rate, until the target heart rate was reached, according to American College of Sport Medicine guidelines. The program began with 10 min of stretching and was conducted using the maximal heart rate index (HRmax) estimated by: 220age. First 2 weeks $=60-70 \%$ of HRmax, 3rd to 12 th weeks $=70-80 \%$ of HRmax [24].

\section{Resistance exercise training}

Patients in Group B were submitted to a 40 min session of resistance training. The program began with $10 \mathrm{~min}$ of stretching and was conducted with exercises done on nine resistance machines. The resistance machines used were: chest press, bicep curl, triceps extension, lower back, abdominals, leg press, leg curl and leg extension.
Subjects performed three sets of $8-12$ repetitions, with $60 \mathrm{~s}$ of rest between each set. Resistance was increased by five pounds after the subject was able to complete three sets of eight repetitions on three consecutive days. Subjects were trained using between 60 and $80 \%$ of their one maximal repetition weight (1-RM) [25].

\section{Statistical analysis}

The mean values of TNF- $\alpha$, IL-6, IL-8, AST and ALT obtained before and after three months in both groups were compared using paired " $\mathrm{t}$ " test. Independent " $\mathrm{t}$ " test was used for the comparison between the two groups $(\mathrm{P}<0.05)$.

\section{Results}

The two groups were considered homogeneous regarding the Baseline descriptive

characteristics (Table 1).

Table (1): comparison of baseline characteristics between patients on mild and moderate exercise.

\begin{tabular}{|c|c|c|c|}
\hline \multirow[b]{2}{*}{ Variable } & \multicolumn{2}{|c|}{ Mean \pm SD } & \multirow[b]{2}{*}{$\mathrm{p}$ value } \\
\hline & $\begin{array}{c}\text { Aerobic exercise } \\
\text { Group (A) }\end{array}$ & $\begin{array}{c}\text { Resistance exercise } \\
\text { Group (B) }\end{array}$ & \\
\hline Age (year) & $50.87 \pm 5.93$ & $51.12 \pm 5.58$ & $\mathrm{P}>0.05$ \\
\hline Weight (Kg) & $93.15 \pm 6.51$ & $92.43 \pm 6.83$ & $\mathrm{P}>0.05$ \\
\hline Height $(\mathrm{cm})$ & $168.21 \pm 7.61$ & $169.35 \pm 6.54$ & $\mathrm{P}>0.05$ \\
\hline $\mathrm{TNF}-\alpha(\mathrm{pg} / \mathrm{mL})$ & $7.76 \pm 1.84$ & $7.89 \pm 1.98$ & $\mathrm{P}>0.05$ \\
\hline IL-6 (pg/mL) & $8.42 \pm 1.67$ & $8.65 \pm 1.67$ & $\mathrm{P}>0.05$ \\
\hline IL-8 (pg/mL) & $18.37 \pm 3.78$ & $18.62 \pm 3.53$ & $\mathrm{P}>0.05$ \\
\hline $\operatorname{ALT}(\mathrm{U} / \mathrm{L})$ & $38.98 \pm 5.34$ & $39.42 \pm 5.87$ & $\mathrm{P}>0.05$ \\
\hline AST (U/L) & $47.65 \pm 6.82$ & $48.16 \pm 6.78$ & $\mathrm{P}>0.05$ \\
\hline
\end{tabular}
TNF- $\alpha=$ tumor necrosis factor - alpha.
IL-6 = Interleukin-6
IL-8= Interleukin-8
ALT $=$ Alanine aminotransferase

$\mathrm{AST}=$ Aspartate aminotransferase

There was a $78.39 \%, 57.67 \%, 49.83 \%, 20.12 \%$ and $15.34 \%$ reduction in mean values of TNF- $\alpha$, IL-6, IL-8, AST and ALT respectively in group (A), however there was a $26.64 \%, 22.17 \%, 20.83 \%, 8.44 \%$ and 6.45
$\%$ reduction in mean values of TNF- $\alpha$, IL-6, IL-8, AST and ALT respectively in group (B) at the end of the study. The mean values of TNF- $\alpha$, IL- 6 , IL- 8 , AST and ALT were significantly decreased in both groups (Table 2 and 3). 
Table (2): Mean value and significance of TNF- $\alpha$, IL-6, IL-8, AST and ALT in group (A) before and after treatment.

\begin{tabular}{|l|c|c|c|c|}
\hline \multirow{2}{*}{} & \multicolumn{2}{|c|}{ Mean \pm SD } & T-value & P-value \\
\cline { 2 - 3 } & Before & After & & \\
\hline TNF- $\alpha(\mathrm{pg} / \mathrm{mL})$ & $7.76 \pm 1.84$ & $4.35 \pm 1.21$ & 5.35 & $\mathrm{P}<0.05$ \\
\hline IL-8 $(\mathrm{pg} / \mathrm{mL} / \mathrm{mL})$ & $8.42 \pm 1.67$ & $5.34 \pm 1.32$ & 5.82 & $\mathrm{P}<0.05$ \\
\hline ALT $(\mathrm{U} / \mathrm{L})$ & $18.37 \pm 3.78$ & $12.26 \pm 2.91$ & 7.84 & $\mathrm{P}<0.05$ \\
\hline AST $(\mathrm{U} / \mathrm{L})$ & $38.98 \pm 5.34$ & $32.45 \pm 4.12$ & 8.72 & $\mathrm{P}<0.05$ \\
\hline
\end{tabular}

TNF- $\alpha=$ tumor necrosis factor - alpha.

IL-6 = Interleukin-6

IL-8= Interleukin-8

ALT $=$ Alanine aminotransferase

AST $=$ Aspartate aminotransferase

Table (3): Mean value and significance of TNF- $\alpha$, IL-6, IL-8, AST and ALT in group (B) before and after treatment.

\begin{tabular}{|l|c|c|c|c|}
\hline \multirow{2}{*}{ TNF- $\alpha(\mathrm{pg} / \mathrm{mL})$} & \multicolumn{2}{|c|}{ Mean \pm T-value } & P-value \\
\cline { 2 - 3 } & Before & After & & \\
\hline IL-6 $(\mathrm{pg} / \mathrm{mL})$ & $8.65 \pm 1.98$ & $6.23 \pm 1.71$ & 3.15 & $\mathrm{P}<0.05$ \\
\hline IL-8 $(\mathrm{pg} / \mathrm{mL})$ & $18.62 \pm 3.53$ & $7.08 \pm 1.23$ & 3.09 & $\mathrm{P}<0.05$ \\
\hline ALT $(\mathrm{U} / \mathrm{L})$ & $39.42 \pm 5.87$ & $36.35 \pm 4.18$ & 4.97 & $\mathrm{P}<0.05$ \\
\hline AST $(\mathrm{U} / \mathrm{L})$ & $48.16 \pm 6.78$ & $45.24 \pm 6.15$ & 5.16 & $\mathrm{P}<0.05$ \\
\hline
\end{tabular}

TNF- $\alpha=$ tumor necrosis factor - alpha.

IL-6 = Interleukin-6

IL-8= Interleukin-8

ALT $=$ Alanine aminotransferase

AST $=$ Aspartate aminotransferase

Also, there were significant differences between mean levels of the investigated parameters in group (A) and group (B) at the end of the study (Table 4) $(\mathrm{P}<0.05)$. 
Table (4): Mean value and significance of TNF- $\alpha$, IL-6, IL-8, AST and ALT in group (A) and group (B) after treatment.

\begin{tabular}{|l|c|c|c|c|}
\hline \multirow{2}{*}{} & \multicolumn{2}{|c|}{ Mean \pm SD } & \multirow{2}{*}{ T-value } & P-value \\
\cline { 2 - 3 } & Group (A) & Group (B) & & \\
\hline TNF- $\alpha(\mathrm{pg} / \mathrm{mL})$ & $4.35 \pm 1.21$ & $6.23 \pm 1.71$ & 3.34 & $\mathrm{P}<0.05$ \\
\hline $\mathrm{IL}-6(\mathrm{pg} / \mathrm{mL})$ & $5.34 \pm 1.32$ & $7.08 \pm 1.23$ & 3.45 & $\mathrm{P}<0.05$ \\
\hline $\mathrm{IL}-8(\mathrm{pg} / \mathrm{mL})$ & $12.26 \pm 2.91$ & $15.41 \pm 3.18$ & 3.82 & $\mathrm{P}<0.05$ \\
\hline ALT $(\mathrm{U} / \mathrm{L})$ & $32.45 \pm 4.12$ & $36.35 \pm 4.84$ & 3.13 & $\mathrm{P}<0.05$ \\
\hline AST $(\mathrm{U} / \mathrm{L})$ & $41.31 \pm 5.33$ & $45.24 \pm 6.15$ & 3.60 & $\mathrm{P}<0.05$ \\
\hline
\end{tabular}

TNF- $\alpha=$ tumor necrosis factor - alpha.

IL-6 = Interleukin-6

IL-8= Interleukin-8

ALT $=$ Alanine aminotransferase

AST $=$ Aspartate aminotransferase

\section{Discussion}

Non-alcoholic steatohepatitis is a common liver disease that can progress to cirrhosis. Currently, there is no established treatment for this disease. So, there is an urgent need for efficient therapeutic options as there is still no approved medication. Moreover, the type, dose and intensity of physical activity needed to obtain a significant anti-inflammatory effect in this high-risk population are largely unknown. Therefore, this study aimed to compare the impact of aerobic and resisted exercise training on inflammatory cytokines and markers of liver function in subjects with nonalcoholic steatohepatitis. The mean values of TNF- $\alpha$, IL-6, IL- 8 , AST and ALT were significantly decreased in group (A) received aerobic exercise training and group (B) received resisted exercise training. Also; there was a significant difference between both groups after treatment, these findings are supported and agreed by several previous studies.

Zhang etal., conducted a study on 28 male SpragueDawley strain rats were randomly assigned into four groups: standard diet without and with concurrent swimming exercise; and high-fat diet without and with concurrent swimming exercise for 12 weeks. The initial swimming duration of 30 minutes was progressively increased by $10 \mathrm{~min} \cdot \mathrm{d}-1$ to 90 minutes by the end of the fourth week, which then remained unchanged, findings revealed that long-term exercise carried out concurrently with consumption of a highfat diet could alleviate high-fat diet-induced NASH which is attributable to the attenuation of hepatic TNF- $\alpha$ overexpression and the upregulation of hepatic peroxisome proliferator activated receptoralpha (PPAR $\alpha)$ mRNA expression [26].

Maintaining or increasing physical activity provides health benefits for patients with fatty liver, independent of changes in weight [27]. Weight, blood biochemical data as aminotransferase, albumin, cholinesterase, total cholesterol and fasting blood glucose values were significantly decreased after a program of restricted diet and exercise (walking or jogging) for a trial period of 3 months in a previous study on twenty-five obese patients with fatty liver [28]. Regular exercise was also associated with significantly greater ALT improvement and normalization. For instance, exercise is known to improve the sensitivity of muscle mass to insulin [29].

The potential mechanisms for the anti-inflammatory effect of exercise include reduced percentage of body fat and macrophage accumulation in adipose tissue, muscle-released interleukin- 6 inhibition of tumour necrosis factor-a, and the cholinergic anti-inflammatory pathway [30].

Eighty-two patients were randomized into 4 groups: sedentary control (A) received low-intensity aerobic exercise, group (B) performed high-intensity aerobic, group $(\mathrm{C})$ performed aerobic and resistance exercises and group (D) performed exercise (with the same caloric expenditure) for 12 months. Physical exercise in type 2 diabetic patients with the metabolic syndrome is associated with a significant reduction of inflammatory and insulin resistance biomarkers, independent of weight loss. Long-term high-intensity (preferably mixed) training, in addition to daytime physical activity, is required to obtain a significant anti-inflammatory effect [31].

It is possible that aerobic exercise decreases subclinical, chronic inflammation and improves endothelial function simply as a result of reducing obesity (particularly 
visceral obesity) and improving insulin sensitivity [32]. During regular aerobic exercise, skeletal muscle fibres inhibit the production of the pro-inflammatory cytokine TNF- $\alpha$ and produce several anti-inflammatory cytokines (termed "myokines") that may be involved in mediating the health-beneficial effects of exercise [33]. Additionally, exercise training may reduce mononuclear cell production of pro-inflammatory cytokines by reducing chronic oxidative stress [34]. Exercise has the potential to lower the inflammatory status by the reduction of tumor necrosis factor $(\mathrm{TNF}-\alpha)[35,36]$.

Exercise suppresses the production of proinflammatory cytokines and enhances anti-inflammatory cytokines. Because proinflammatory cytokines, IL- 6 and TNF- $\alpha$, have cytotoxic actions, it can be proposed that regular exercise prevents further damage to insulin-producing $\beta$-cells by attenuating the production of these cytokines $[33,37]$. However, high-intensity exercise training program induced an improvement of biomarkers of inflammation and insulin resistance, with a reduction of IL-6, TNF- $\alpha$ and leptin and an increase of IL-4 and IL-10--- thus indicating that exercise has a full antiinflammatory effect [38].

\section{Conclusion}

Aerobic exercise training modulates inflammatory cytokine levels and markers of liver function in patients with nonalcoholic steatohepatitis.

\section{Acknowledgment}

This project was funded by the Deanship of Scientific Research (DSR), King Abdulaziz University, Jeddah, under grant no. (166-142-1433). The authors, therefore, acknowledge with thanks DSR technical and financial support.

\section{References}

1. Angulo P. Nonalcoholic fatty liver disease. N Engl J Med 2002; ;346(16):1221-31.

2. Ji G, Yang Q, Hao J, Guo L, Chen X, Hu J, Leng L, Jiang Z. Anti-inflammatory effect of genistein on nonalcoholic steatohepatitis rats induced by high fat diet and its potential mechanisms . International Immunopharmacology 2011; 11(6): 762-768.

3. McCullough A. Pathophysiology of nonalcoholic steatohepatitis. J Clin Gastroenterol 2006 ;40 Suppl 1:S17-29.

4. Musso G, Gambino R, Cassader M, Pagano G. A meta-analysis of randomized trials for the treatment of nonalcoholic fatty liver disease. Hepatology 2010; 52(1): 79-104.

5. Koek G, Liedorp P, Bast A. The role of oxidative stress in non-alcoholic steatohepatitis. Clinica Chimica Acta 2011; 412(15-16):1297-1305.

6. Harrison S, Kadakia S, Lang K, Schenker S. Nonalcoholic steatohepatitis: what we know in the new millennium. Am J Gastroenterol 2002; 97 (11): 2714-2724.

7. Armutcu F, Coskun O, Gurel A, Kanter M, Can M, Ucar F. Thymosin alpha 1 attenuates lipid peroxidation and improves fructose-induced steatohepatitis in rats. Clin Biochem 2005; 38(6): 540-547.

8. Houstis N, Rosen E, Lander E. Reactive oxygen species have a causal role in multiple forms of insulin resistance. Nature 2006; 440(7086):944-8.

9. Postic C, Girard J. Contribution of de novo fatty acid synthesis to hepatic steatosis and insulin resistance: lessons from genetically engineered mice. J Clin Invest 2008; 118(3): 829-838.

10. Uysal S, Armutcu F, Aydogan T, Akin K, Ikizek M, Yigitoglu M. Some inflammatory cytokine levels, iron metabolism and oxidan stress markers in subjects with nonalcoholic steatohepatitis.Clinical Biochemistry 2011; 44(17-18): 1375-1379.

11. Schindhelm R, Diamant M, Dekker J, Tushuizen M, Teerlink T, Heine R. Alanine aminotransferase as a marker of non-alcoholic fatty liver disease in relation to type 2 diabetes mellitus and cardiovascular disease. Diabetes Metab Res Rev 2006; 22(6): 437-443.

12. Lewis J, Mohanty S. Nonalcoholic fatty liver disease: a review and update. Dig Dis Sci 2010; 55(3): 560-578. 13. Garg A, Misra A. Hepatic steatosis, insulin resistance, and adipose tissue disorders. J Clin Endocrinol Metab 2002; 87(7): 3019-3022.

14. Xu A, Wang Y, Keshaw H, Xu L, Lam K, Cooper G. The fat-derived hormone adiponectin alleviates alcoholic and nonalcoholic fatty liver diseases in mice. J Clin Invest 2003; 112 (1): 91-100.

15. Day C. Pathogenesis of steatohepatitis. Best Pract Res Clin Gastroenterol 2002; 16 (5): 663-678.

16. Misra A, Vikram N. Clinical and pathophysiological consequences of abdominal adiposity and abdominal adipose tissue depots. Nutrition 2003; 19 (5): 457-466. 17. Hui JM, Hodge A, Farrell GC, Kench JG, Kriketos A, George J. Beyond insulin resistance in NASH: TNFalpha or adiponectin?. Hepatology 2004;40(1):46-54.

18. Targher G, Bertolini L, Scala L, Poli F, Zenari L, Falezza G. Decreased plasma adiponectin concentrations are closely associated with nonalcoholic hepatic 
steatosis in obese individuals. Clin Endocrinol (Oxf). 2004;61(6):700-3.

19. Schäffler A, Schölmerich J, Büchler C. Mechanisms of disease: adipocytokines and visceral adipose tissue-emerging role in nonalcoholic fatty liver disease. Nat Clin Pract Gastroenterol Hepatol. 2005 ;2(6):273-80.

20. Oberbach A, Tönjes A, Klöting N, Fasshauer M, Kratzsch J, Busse MW, Paschke R, Stumvoll M, Blüher M. Effect of a 4 week physical training program on plasma concentrations of inflammatory markers in patients with abnormal glucose tolerance. Eur J Endocrinol 2006; 154 (4):577-585.

21. Zoppini G, Targher G, Zamboni C, Venturi C, Cacciatori V, Moghetti P, Muggeo M. Effects of moderate-intensity exercise training on plasma biomarkers of inflammation and endothelial dysfunction in older patients with type 2 diabetes. Nutr Metab Cardiovasc Dis Oberbach 2006; 16 (8): 543-549.

22. Woods J, Vieira V, Todd K. Exercise, Inflammation, and Innate Immunity. Immunology and Allergy Clinics of North America 2009; 29(2):381-393.

23. Saadeh S, Younossi Z, Remer E, Gramlich T, Ong $\mathrm{J}$, Hurley $\mathrm{M}$. The utility of radiological imaging in nonalcoholic fatty liver disease. Gastroenterology 2002; 123(3): 745-750.

24. Robergs RA, Landwehr R. The surprising history of the "HRmax $=220$-age" equation. J Exerc Physiol Online 2002; 5(2):1-10.

25. Ramalho AC, de Lourdes Lima M, Nunes F, Cambuí Z, Barbosa C, Andrade A, Viana A, Martins M, Abrantes V, Aragão C, Temístocles M. The effect of resistance versus aerobic training on metabolic control in patients with type- 1 diabetes mellitus. Diabetes Res Clin Pract 2006; 72(3):271-6.

26. Zhang H, He Y, Chung P, Tong T, Fu F, Chen Y, Jiao G. Effects of 12 Weeks of Exercise on Hepatic TNF- $\alpha$ and PPAR $\alpha$ in an Animal Model of High-Fat Diet-Induced Nonalcoholic Steatohepatitis. Journal of Exercise Science \& Fitness 2009; 7(1):18-23.

27. St George A, Bauman A, Johnston A. Independent effects of physical activity in patients with nonalcoholic fatty liver disease. Hepatology.2009; 50 (1): 68-76.

28. Ueno T, Sugawara H, Sujaku K, Hashimoto O, Tsuji
R, Tamaki S, Torimura T, Inuzuka S, Sata M, Tanikawa K.Therapeutic effects of restricted diet and exercise in obese patients with fatty liver. Journal of Hepatology1997; 27(1):103-107.

29. Perseghin G, Price T, Petersen K, Roden M, Cline G , Gerow K. Increased glucose transport-phosphorylation and muscle glycogen synthesis after exercise training in insulin-resistant subjects. N Engl J Med 1996; 335: 1357-1362.

30. Woods JA, Vieira VJ, Keylock KT. Exercise, inflammation, and innate immunity. Neurol Clin 2006; 24(3):585-99.

31. Balducci S, Zanuso S, Nicolucci A, Fernando F, Cavallo S, Cardelli P, Fallucca S. Anti-inflammatory effect of exercise training in subjects with type 2 diabetes and the metabolic syndrome is dependent on exercise modalities and independent of weight loss. Nutrition, Metabolism and Cardiovascular Diseases 2010; 20(8): 608-617.

32. Hu G, Jousilahti P, Barengo N, Qiao Q, Lakka T, Tuomilehto J. Physical activity, cardiovascular risk factors, and mortality among Finnish adults with diabetes. Diabetes Care 2005; 28(4):799-805.

33. Petersen AM, Pedersen BK. The anti-inflammatory effect of exercise. J Appl Physiol 2005; 98(4): 11541162.

34. Stewart K. Role of exercise training on cardiovascular disease in persons who have type 2 diabetes and hypertension. Cradiol Clin 2004; 22(4): 569-86.

35. Mathur N, Pedersen BK. Exercise as a mean to control low-grade systemic inflammation. Mediators Inflamm. 2008;2008:109502.

36. Monzillo LU, Hamdy O, Horton ES. Effect of lifestyle modification on adipokine levels in obese subjects with insulin resistance. Obes Res 2003; 11(9):1048 1054.

37. Smith J, Dykes R, Douglas J, Krishnaswamy G, Berk $\mathrm{S}$. Long-term exercise and atherogenic activity of blood mononuclear cells in persons at risk of developing ischemic heart disease. JAMA 1999; 281(18):1722-7.

38. Calabro P, Chang D, Willerson J, Yeh E. Release of C-reactive protein in response to inflammatory cytokines by human adipocytes: linking obesity to vascular inflammation. J Am Coll Cardiol 2005; 46(6):11121113. 